\title{
Screening of the Growth-Inhibitory Effects of 168 Plant Species against Lettuce Seedlings
}

\author{
Tomoko Takemura, Emi Sakuno, Tsunashi Kamo*, Syuntaro Hiradate, Yoshiharu Fujii
}

Biodiversity Division, National Institute for Agro-Environmental Sciences, Tsukuba, Japan.

Email: *tkamo@affrc.go.jp

Received March $9^{\text {th }}, 2013$; revised April 15 ${ }^{\text {th }}, 2013$; accepted May $10^{\text {th }}, 2013$

Copyright (C) 2013 Tomoko Takemura et al. This is an open access article distributed under the Creative Commons Attribution License, which permits unrestricted use, distribution, and reproduction in any medium, provided the original work is properly cited.

\begin{abstract}
The methanol extracts of 168 plant species from 68 families were evaluated for their inhibitory activity against lettuce seedling elongation. Among the plant species tested, 12 species had $\mathrm{EC}_{50}$ values for radicle growth inhibition ranging from 0.01 to $5.00 \mathrm{mg}$ fresh weight equivalent $\mathrm{mL}^{-1}$. Enterolobium contortisiliquum, a traditionally used herbal medicine, exhibited the strongest inhibitory activity (estimated $\mathrm{EC}_{50}$ : 0.28 fresh weight equivalent $\mathrm{mL}^{-1}$ ). Among the 12 species, Pachysandra terminalis, Tamarindus indica, and Albizia guachapele required investigation, because only little has been reported about their chemical constituents to date. The data in the present study would be useful in finding new lead compounds for natural herbicides.
\end{abstract}

Keywords: Screening; $\mathrm{EC}_{50}$; Allelochemical; Plant Growth Inhibition

\section{Introduction}

Weed control is a major problem in agriculture. Uncontrolled weeds cause enormous reductions in crop yields $[1,2]$. Synthetic herbicides play an important role in weed suppression [3], but the overuse of chemical fertilizers and agrochemicals poses many risks to agricultural fields and our environment such as desertification, soil salinization, and the pollution of ground water [4,5]. Natural compounds have the potential to partially replace synthetic herbicides or serve as starting materials for the chemical synthesis of biodegradable herbicides, considering that they should be less harmful to the environment than synthetic herbicides owing to their simple degradation. Allelochemicals (natural plant toxins that play a role in plantplant, plant-insect, and plant-microorganism interactions) are a promising source of new herbicides for weed control because they can potentially have more specific interactions with target organisms and cause less environmental damage [6-8]. A prominent example of an effective allelochemical is the case of leptospermone, a natural sesquiterpene found in the lemon bottlebrush Callistemon citrinus [9]. Mesotrione, a structurally modified analog of leptospermone, exhibits far stronger inhibitory activity against 4-hydroxyphenylpyruvate dioxygenase than the lead compound [10]. Currently, an agricultural chemical containing

${ }^{*}$ Corresponding author. mesotrione is on the market.

In the present study, we evaluated the plant growth-inhibitory activity of the methanol extracts of 168 plant species to estimate their potential as sources of bioactive compounds. While allelopathy was the main purpose of the screening studies reported by our laboratory [11-13], we intended to identify potential species for the isolation and characterization of plant growth-inhibitory compounds in the present work; therefore, plant extracts were used for bioassays instead of living plants or dried leaves [14, 15]. To investigate the active compounds in the potential species, the availability of plant material was considered the top priority when the species were selected. Therefore, we collected the leaves of weeds and ornamental plants that are common in the Kanto region of Japan together with those of herbaceous plants and trees grown in a botanical garden. The results allowed us to estimate the inhibitory potential of various species, leading to the application of plant growth-inhibitory compounds in weed management for sustainable agriculture.

\section{Materials and Methods}

\subsection{Plant Materials}

The aerial parts of common weeds and ornamental plants were collected in Matsudo and Tsukuba, Japan. Those of tropical and subtropical plants were sampled in green- 
houses of the National Institute for Agro-Environmental Sciences, Tsukuba, Japan and Tsukuba Botanical Garden, National Museum of Nature and Science, Tsukuba, Japan. The plant materials were immediately soaked in methanol and left at room temperature for 2 weeks. The methanol extracts were used for bioassays after filtration.

\subsection{Bioassay}

Filter paper (27 mm ø, Toyo Roshi Kaisha, Ltd, Tokyo) was placed in a glass Petri dish $(27 \mathrm{~mm} \varnothing)$. Test solution was added to the filter paper in the Petri dish and dried completely in vacuo. After adding distilled water (0.7 $\mathrm{ml})$, five pregerminated $\left(20 \mathrm{~h}\right.$ at $20^{\circ} \mathrm{C}$ in the dark) seedlings of lettuce (Lactuca sativa cv. Great Lakes 366) were placed on the filter paper and incubated for $52 \mathrm{~h}$ at $20^{\circ} \mathrm{C}$ in the dark. The final concentration ranged from 0.43 to $1429 \mathrm{mg}$ fresh weight equivalent $\mathrm{mL}^{-1}$ (F.W. eq. $\mathrm{mL}^{-1}$ ). Inhibition of radicle elongation was determined by comparing the radicle lengths of treated plants with that of control plants. The effective concentrations required to induce half-maximal inhibition of growth $\left(\mathrm{EC}_{50}\right)$ and $95 \%$ confidence intervals were calculated by the probit method using SPSS for Windows statistical software (ver. 11.0.1J, IBM, Armonk, NY, USA).

\section{Results and Discussion}

The estimated $\mathrm{EC}_{50}$ values of 168 plant species against elongation of the radicles of lettuce seedlings are shown in Table 1. $\mathrm{EC}_{50}$ means the effective concentration of a compound to induce half-maximum action, which is defined as the concentration of agonist that provokes a response half way between the baseline and maximum response. Species were categorized according to their $\mathrm{EC}_{50}$ values: 0.01 - $5.00 \mathrm{mg}$ F.W. eq. $\mathrm{mL}^{-1}, 12$ species; 5.01 - 10.0 mg F.W. eq. $\mathrm{mL}^{-1}, 26$ species; $10.1-20.0 \mathrm{mg}$ F.W. eq. $\mathrm{mL}^{-1}, 41$ species; 20.1 - $50.0 \mathrm{mg}$ F.W. eq. $\mathrm{mL}^{-1}$, 58 species; and 50.1 - $400 \mathrm{mg}$ F.W. eq. $\mathrm{mL}^{-1}, 31$ species.

In this section, we describe the physiological and chemical information of the 12 species with $\mathrm{EC}_{50}$ values of elongation inhibition against lettuce radicle of 0.01 $5.00 \mathrm{mg} \mathrm{F.W}$. eq. $\mathrm{mL}^{-1}$. Enterolobium contortisiliquum (Mimosaceae) displayed the strongest inhibitory activity (estimated $\mathrm{EC}_{50}: 0.28 \mathrm{~F} . \mathrm{W}$. eq. $\mathrm{mL}^{-1}$ ) among the 168 species tested in the present study. It is a herbal medicine traditionally used to treat parasitism and gonorrhea in Brazil [16]. Mimaki et al. [17] reported that the pericarps of this species contained bisdesmosidic triterpene saponins and that some of these compounds exhibited cytotoxicity. We conducted partial isolation of the active compounds from the crude extract of E. contortisiliquum. ${ }^{1} \mathrm{H}-\mathrm{NMR}$ analysis suggested that the fraction exhibiting major plant growth inhibitory activity consists of a mix- ture of triterpene saponins (data not shown).

Spiraea thunbergii (Rosaceae) displayed the second strongest inhibitory activity (estimated $\mathrm{EC}_{50}: 0.32 \mathrm{~F} . \mathrm{W}$. eq. $\mathrm{mL}^{-1}$ ). We selected this species as a positive control because it is already established that $S$. thunbergii exhibits strong plant growth-inhibitory activity [18]. This is due to two major bioactive compounds possessing a ciscinnamoyl moiety, 1-O-cis-cinnamoyl- $\beta$-D-glucopyranose and 6-O-(4'-hydroxy-2'-methylenebutyroyl)-1-O-cis-cinnamoyl- $\beta$-D-glucopyranose [19].

Convallaria keiskei (Liliaceae) had an estimated $\mathrm{EC}_{50}$ value of 0.87 F.W. eq. $\mathrm{mL}^{-1}$. Convallaria species produce white flowers with a mild fragrance and contain cardiac glycosides; therefore, many studies have been performed to evaluate the horticultural, medical, chemical, and physiological properties of this genus [20-22]. However, the plant growth-inhibitory activity of this species has not been reported thus far.

Gliricidia sepium (Fabaceae; estimated $\mathrm{EC}_{50}: 1.78$ F.W. eq. $\mathrm{mL}^{-1}$ ) is distributed throughout seasonal dry forests in Mexico and other Central American countries [23]. This plant is used for living fences, fodder for ruminants, green manure, shade, and firewood in addition to its use as a rodenticide [24]. Ramamoorthy and Paliwal [25] reported fifteen phenolics from the leaves of this species as allelochemicals. Based on the present screening study, we recently isolated the compound most strongly contributing to the inhibitory activity of $G$. sepium and identified this compound as coumarin. This finding will be published elsewhere.

Pachysandra terminalis (Buxaceae; estimated $\mathrm{EC}_{50}$ : 1.92 F.W. eq. $\mathrm{mL}^{-1}$ ) is a small shrub (approximately 30 $\mathrm{cm}$ in height) distributed in Japan. It is used as a decorative plant, and the dried plant has been used as folk medicine for stomachache in the northern part of Japan [26]. It exhibits strong cytotoxic activity against P388 mouse leukemia cells [27]. Allelopathic potential, if it exists, might contribute to the dominance that $P$. terminalis often displays in the habitat.

Samanea saman (Mimosaceae; estimated $\mathrm{EC}_{50}: 2.20$ F.W. eq. $\mathrm{mL}^{-1}$ ) is native to tropical America. It has now become widespread throughout the humid and sub-humid tropics. Commonly referred to as "monkey pod" or "cow tamarind," this plant is traditionally used to treat diarrhea, intestinal diseases, stomach aches, colds, and headaches [28]. The leaves of $S$. saman also display antimicrobial activity [29]. The leaves were reported to constitute tannins, flavonoids, steroids, saponins, cardiac glycosides, and terpenoids [28]. Although the allelopathic potential of this plant was reported [30], the allelochemicals were not elucidated.

Tamarindus indica (Caesalpiniaceae) exhibited strong 
Table 1. $\mathrm{EC}_{50}$ values of the crude extracts from the aerial parts of 168 plant species.

\begin{tabular}{|c|c|c|c|}
\hline Family & Scientific name & $\mathrm{EC}_{50}$ value $^{1}$ & Sampling location ${ }^{2}$ \\
\hline Agavaceae & Cordyline teriminalis & $38.0(25.7-56.9)$ & $\mathrm{c}$ \\
\hline Aloaceae & Aloe vera & $331(253-489)$ & d \\
\hline Amaranthaceae & Alternanthera denticulate & $29.1(20.6-41.1)$ & $\mathrm{c}$ \\
\hline Amaryllidaceae & Clivia miniata & $10.4(5.55-18.6)$ & $\mathrm{d}$ \\
\hline Annonaceae & Asimina triloba & $13.9(7.45-24.9)$ & a \\
\hline \multirow[t]{3}{*}{ Apiaceae } & Angelica keiskei & $104(88.8-125)$ & $\mathrm{c}$ \\
\hline & Chamaele decumbens & $90.7(41.3-238)$ & a \\
\hline & Cicuta virosa & $278(240-321)$ & $\mathrm{c}$ \\
\hline Apocynaceae & Catharanthus roseus & $114(95.7-135)$ & $\mathrm{d}$ \\
\hline Aquifoliaceae & Ilex crenata var. crenata & $31.2(21.5-45.5)$ & d \\
\hline \multirow[t]{3}{*}{ Araceae } & Arisaema thunbergii subsp. urashima & $84.8(56.3-157)$ & d \\
\hline & Pinellia ternata & $33.8(48.4-70.2)$ & $\mathrm{d}$ \\
\hline & Pistia stratiotes & $35.3(29.2-42.7)$ & $\mathrm{c}$ \\
\hline \multirow[t]{2}{*}{ Araliaceae } & Dendropanax trifidus & $83.0(67.3-102)$ & a \\
\hline & Hedera rhombea & $12.2(9.14-16.1)$ & d \\
\hline Asclepiadaceae & Metaplexis japonica & $29.6(18.3-48.8)$ & d \\
\hline \multirow[t]{13}{*}{ Asteraceae } & Cynara scolymus & $91.0(75.5-112)$ & $\mathrm{a}$ \\
\hline & Dahlia imperialis & $44.2(30.0-66.8)$ & d \\
\hline & Erigeron philadelphicus & $42.3(33.2-52.6)$ & $\mathrm{b}$ \\
\hline & Farfugium japonicum & $237(181-309)$ & $\mathrm{d}$ \\
\hline & Galinsoga quadriradiata & $18.8(10.3-34.6)$ & $\mathrm{b}$ \\
\hline & Helianthus maximiliani & $66.7(54.3-81.7)$ & $\mathrm{c}$ \\
\hline & Petasites japonicus var. japonicus & $219(189-253)$ & a \\
\hline & Picris hieracioides subsp. japonica & $25.2(15.8-40.8)$ & $\mathrm{b}$ \\
\hline & Senecio jacobaea & $107(80.2-137)$ & $\mathrm{c}$ \\
\hline & Senecio kleiniiformis & $36.0(26.4-50.0)$ & a \\
\hline & Solidago altissima & $146(125-172)$ & $\mathrm{b}$ \\
\hline & Stenactis annuиs & $25.5(14.6-45.6)$ & $\mathrm{b}$ \\
\hline & Taraxacum officinale & $31.6(22.3-45.1)$ & $\mathrm{b}$ \\
\hline \multirow[t]{2}{*}{ Berberidaceae } & Epimedium grandiflorum & $25.7(14.8-44.7)$ & d \\
\hline & Nandina domestica & $6.21(4.61-8.30)$ & $\mathrm{d}$ \\
\hline Boraginaceae & Symphytum officinale & $218(157-311)$ & $\mathrm{c}$ \\
\hline Buxaceae & Pachysandra terminalis & $1.92(1.21-2.82)$ & $\mathrm{c}$ \\
\hline \multirow[t]{3}{*}{ Caesalpiniaceae } & Caesalpinia pluviosa var. peltophroides & $3.76(2.96-4.72)$ & $\mathrm{c}$ \\
\hline & Senna obtusifolia & $18.5(12.3-28.0)$ & $\mathrm{c}$ \\
\hline & Tamarindus indica & $2.51(1.36-4.13)$ & $\mathrm{c}$ \\
\hline Calycanthaceae & Calycanthus floridus var. floridus & $8.23(5.52-12.1)$ & d \\
\hline Campanulaceae & Triodanis perfoliata & $124(52.2-373)$ & $\mathrm{b}$ \\
\hline
\end{tabular}




\section{Continued}

\begin{tabular}{|c|c|c|c|}
\hline Cannaceae & Canna edulis & $197(133-261)$ & $\mathrm{c}$ \\
\hline Capparaceae & Crateva formosensis & $41.5(25.7-68.9)$ & $\mathrm{c}$ \\
\hline \multirow[t]{4}{*}{ Caprifoliaceae } & Abelia $\times$ grandiflora & $49.5(33.8-72.8)$ & $\mathrm{d}$ \\
\hline & Lonicera japonica & $35.4(25.2-50.4)$ & $\mathrm{d}$ \\
\hline & Lonicera morrowii & $33.1(12.3-93.1)$ & $\mathrm{a}$ \\
\hline & Weigela coraeensis & $132(112-155)$ & d \\
\hline \multirow[t]{2}{*}{ Celastraceae } & Celastrus orbiculatus var. orbiculatus & $62.6(47.3-87.7)$ & $\mathrm{b}$ \\
\hline & Euonymus fortunei & $20.3(12.9-32.1)$ & $\mathrm{a}$ \\
\hline Chloranthaceae & Sarcandra glabra & $18.5(12.0-25.4)$ & $\mathrm{d}$ \\
\hline Cornaceae & Aucuba japonica var. japonica & $19.5(15.5-24.6)$ & $\mathrm{d}$ \\
\hline Crassulaceae & Phedimus aizoon var. floribundus & $48.4(35.1-65.7)$ & $\mathrm{c}$ \\
\hline \multirow[t]{3}{*}{ Cupressaceae } & Juniperus conferta & $22.9(11.9-44.8)$ & a \\
\hline & Thujopsis dolabrata var. hondae & $16.0(12.2-20.8)$ & $\mathrm{c}$ \\
\hline & Thujopsis dolabrata & $9.00(5.74-13.8)$ & $\mathrm{b}$ \\
\hline Cycadaceae & Cycas revoluta & $26.3(19.5-34.8)$ & $\mathrm{c}$ \\
\hline Davidiaceae & Davidia involucrata & $12.4(9.23-16.7)$ & $\mathrm{a}$ \\
\hline Dryopteridaceae & Cyrtomium fortunei var. clivicola & $12.4(8.35-18.4)$ & $\mathrm{b}$ \\
\hline Equisetaceae & Equisetum arvense & $40.4(31.1-51.9)$ & $\mathrm{b}$ \\
\hline \multirow[t]{3}{*}{ Ericaceae } & Kalmia latifolia & $4.79(2.30-9.41)$ & $\mathrm{d}$ \\
\hline & Pieris japonica & $10.4(7.23-14.9)$ & d \\
\hline & Rhododendoron sp. & $7.46(4.82-11.3)$ & $\mathrm{d}$ \\
\hline \multirow[t]{3}{*}{ Euphorbiaceae } & Bischofia javanica & $7.14(4.80-10.7)$ & $\mathrm{c}$ \\
\hline & Euphorbia lasiocaula & $26.5(17.9-39.2)$ & $\mathrm{b}$ \\
\hline & Euphorbia platyphyllos & $37.2(25.8-54.1)$ & $\mathrm{c}$ \\
\hline \multirow[t]{15}{*}{ Fabaceae } & Acacia podalyriifolia & $4.75(3.02-6.65)$ & $\mathrm{a}$ \\
\hline & Albizia guachapele & $3.08(2.43-3.93)$ & $\mathrm{c}$ \\
\hline & Astragalus sinicus & $20.1(16.6-24.9)$ & $\mathrm{b}$ \\
\hline & Cassia ferruginea & $6.21(3.59-10.3)$ & $\mathrm{c}$ \\
\hline & Castanopsis sieboldii & $11.3(7.62-16.8)$ & $\mathrm{b}$ \\
\hline & Erythrina speciosa & $70.8(51.6-90.9)$ & $\mathrm{c}$ \\
\hline & Gliricidia sepium & $1.78(1.19-2.50)$ & $\mathrm{c}$ \\
\hline & Indigofera kirilowii & $3.08(1.99-4.58)$ & $\mathrm{a}$ \\
\hline & Lespedeza bicolor & $37.6(31.5-44.6)$ & $\mathrm{c}$ \\
\hline & Pueraria lobata & $14.8(11.6-18.8)$ & $\mathrm{b}$ \\
\hline & Robinia pseudo-acacia & $16.6(12.8-21.2)$ & $\mathrm{c}$ \\
\hline & Styphnolobium japonicum & $62.5(48.8-78.5)$ & $\mathrm{b}$ \\
\hline & Trifolium pratense & $47.4(34.7-64.9)$ & $\mathrm{b}$ \\
\hline & Vicia unijuga & $22.4(16.8-29.8)$ & $\mathrm{a}$ \\
\hline & Vicia villosa subsp. villosa & $21.1(15.3-29.1)$ & $\mathrm{b}$ \\
\hline
\end{tabular}




\section{Continued}

\begin{tabular}{|c|c|c|c|}
\hline Fabaceae & Wisteria floribunda & $8.87(6.96-11.2)$ & $\mathrm{b}$ \\
\hline Gentianaceae & Centaurium tenuiflorum & $252(215-294)$ & $\mathrm{c}$ \\
\hline \multirow[t]{2}{*}{ Geraniaceae } & Geranium carolinianum & $18.6(14.6-23.3)$ & $\mathrm{d}$ \\
\hline & Pelargonium graveolens & $22.6(15.3-30.5)$ & $\mathrm{c}$ \\
\hline \multirow[t]{2}{*}{ Hamamelidaceae } & Liquidambar styraciflua & $9.61(6.12-15.1)$ & $\mathrm{b}$ \\
\hline & Loropetalum chinense & $9.66(6.18-14.8)$ & $\mathrm{a}$ \\
\hline Iridaceae & Iris pseudacorus & $58.5(42.5-77.2)$ & $\mathrm{c}$ \\
\hline \multirow[t]{2}{*}{ Lamiaceae } & Mentha suaveolens & $107(48.2-195)$ & $\mathrm{c}$ \\
\hline & Salvia elegans & $123(85.6-175)$ & $\mathrm{a}$ \\
\hline \multirow[t]{7}{*}{ Liliaceae } & Allium tuberosum & $13.4(9.54-18.5)$ & $\mathrm{b}$ \\
\hline & Convallaria keiskei & $0.87(0.23-2.03)$ & $\mathrm{d}$ \\
\hline & Disporum sessile & $23.5(18.9-29.0)$ & $\mathrm{d}$ \\
\hline & Eucomis autumnalis & $16.9(8.57-33.5)$ & $\mathrm{a}$ \\
\hline & Reineckea carnea & $13.8(9.42-20.0)$ & $\mathrm{c}$ \\
\hline & Rohdea japonica & $28.0(15.9-49.4)$ & $\mathrm{d}$ \\
\hline & Barnardia japonica & $31.3(25.6-38.2)$ & $\mathrm{d}$ \\
\hline Lythraceae & Cuphea hyssopifolia & $8.57(6.56-10.7)$ & $\mathrm{c}$ \\
\hline Meliaceae & Azadirachta indica & $22.9(14.7-36.4)$ & $\mathrm{c}$ \\
\hline \multirow[t]{4}{*}{ Mimosaceae } & Enterolobium contortisiliquum & $0.28(0.15-0.40)$ & $\mathrm{c}$ \\
\hline & Leucaena leucocephala & $11.8(9.42-14.5)$ & $\mathrm{c}$ \\
\hline & Samanea saman & $2.20(1.14-3.73)$ & $\mathrm{c}$ \\
\hline & Securigera varia & $9.01(5.40-13.4)$ & $\mathrm{c}$ \\
\hline Moringaceae & Moringa oleifera & $92.3(66.4-129)$ & $\mathrm{c}$ \\
\hline \multirow[t]{2}{*}{ Moraceae } & Ficus carica & $9.38(7.29-12.0)$ & $\mathrm{c}$ \\
\hline & Ficus pumila & $40.9(31.0-53.8)$ & $\mathrm{c}$ \\
\hline Myrsinaceae & Ardisia crenata & $7.35(5.41-9.82)$ & $\mathrm{d}$ \\
\hline \multirow[t]{2}{*}{ Myrtaceae } & Callistemon citrinus & $22.7(13.0-40.4)$ & $\mathrm{a}$ \\
\hline & Eucalyptus citriodora & $15.7(12.5-19.8)$ & $\mathrm{c}$ \\
\hline \multirow[t]{5}{*}{ Oleaceae } & Forsythia viridissima & $10.7(7.34-14.6)$ & $\mathrm{a}$ \\
\hline & Ligustrum lucidum & $51.8(42.5-62.6)$ & $\mathrm{a}$ \\
\hline & Osmanthus fragrans var. aurantiacus & $19.7(12.9-31.0)$ & a \\
\hline & Osmanthus heterophyllus & $17.3(12.3-24.2)$ & $\mathrm{d}$ \\
\hline & Syringa vulgaris & $30.5(27.2-34.1)$ & $\mathrm{a}$ \\
\hline Onagraceae & Oenothera stricta & $40.0(26.2-62.3)$ & $\mathrm{b}$ \\
\hline \multirow[t]{2}{*}{ Orchidaceae } & Bletilla striata & $6.61(4.34-9.10)$ & $\mathrm{c}$ \\
\hline & Calanthe discolor & $7.70(6.13-9.64)$ & $\mathrm{a}$ \\
\hline Pandanaceae & Pandanus boninensis & $19.9(15.9-25.0)$ & $\mathrm{b}$ \\
\hline \multirow[t]{2}{*}{ Papaveraceae } & Chelidonium majus var. asiaticum & $17.2(13.4-22.1)$ & $\mathrm{d}$ \\
\hline & Corydalis incisa & $6.90(5.07-9.24)$ & $\mathrm{d}$ \\
\hline
\end{tabular}




\section{Continued}

\begin{tabular}{|c|c|c|c|}
\hline Papaveraceae & Macleaya cordata & $104(83.2-141)$ & $\mathrm{d}$ \\
\hline Pedaliaceae & Uncarina grandidieri & $56.8(47.5-67.5)$ & $\mathrm{a}$ \\
\hline Phytolaccaceae & Phytolacca americana & $66.5(51.6-81.7)$ & $\mathrm{b}$ \\
\hline \multirow[t]{2}{*}{ Pinaceae } & Abies firma & $14.3(7.63-26.7)$ & $\mathrm{a}$ \\
\hline & Cedrus deodara & $7.42(6.04-9.46)$ & $\mathrm{b}$ \\
\hline \multirow[t]{7}{*}{ Poaceae } & Briza maxima & $81.8(65.0-99.6)$ & $\mathrm{b}$ \\
\hline & Bromus catharticus & $20.7(15.2-28.1)$ & $\mathrm{b}$ \\
\hline & Cortaderia selloana & $36.4(23.2-58.6)$ & $\mathrm{b}$ \\
\hline & Eremochloa ophiuroides & $8.48(5.78-12.3)$ & $\mathrm{c}$ \\
\hline & Pennisetum clandestinum & $14.1(11.1-17.9)$ & $\mathrm{c}$ \\
\hline & Phragmites australis & $12.1(8.91-16.3)$ & $\mathrm{c}$ \\
\hline & Sasaella kogasensis var. gracillima & $19.5(13.6-28.0)$ & $\mathrm{c}$ \\
\hline \multirow[t]{2}{*}{ Polygonaceae } & Fallopia japonica var. japonica & $5.13(2.57-7.60)$ & $\mathrm{b}$ \\
\hline & Polygonum perfoliatum & $7.78(6.35-9.28)$ & $\mathrm{d}$ \\
\hline Pontederiaceae & Eichhornia crassipes & $39.8(25.8-62.9)$ & $\mathrm{c}$ \\
\hline \multirow[t]{2}{*}{ Ranunculaceae } & Aquilegia caerulea & $22.0(14.5-33.6)$ & $\mathrm{c}$ \\
\hline & Helleborus niger & $12.3(9.30-15.8)$ & $\mathrm{d}$ \\
\hline \multirow[t]{11}{*}{ Rosaceae } & Cydonia oblonga & $8.62(5.43-13.6)$ & a \\
\hline & Eriobotrya japonica & $22.9(15.4-34.3)$ & $\mathrm{d}$ \\
\hline & Fragaria $\times$ ananassa & $12.3(8.10-18.7)$ & $\mathrm{b}$ \\
\hline & Kerria japonica & $19.0(12.5-28.6)$ & $\mathrm{a}$ \\
\hline & Potentilla chinensis & $26.5(18.2-38.7)$ & $\mathrm{c}$ \\
\hline & Prunus sp. & $18.5(15.2-22.5)$ & $\mathrm{b}$ \\
\hline & Rhaphiolepis indica var. umbellate & $21.3(16.9-25.8)$ & $\mathrm{b}$ \\
\hline & Rubus hirsutus & $23.5(17.4-29.8)$ & a \\
\hline & Sanguisorba minor & $9.52(7.58-11.9)$ & $\mathrm{c}$ \\
\hline & Spiraea cantoniensis & $5.38(3.58-7.87)$ & $\mathrm{d}$ \\
\hline & Spiraea thunbergii & $0.32(0.18-0.47)$ & $\mathrm{b}$ \\
\hline \multirow[t]{5}{*}{ Rutaceae } & Citrus hanayu & $11.5(8.91-14.9)$ & $\mathrm{a}$ \\
\hline & Citrus junos & $29.8(20.6-40.1)$ & $\mathrm{d}$ \\
\hline & Ruta graveolens & $15.3(10.5-22.3)$ & $\mathrm{c}$ \\
\hline & Ptelea trifoliata & $5.95(4.12-8.33)$ & $\mathrm{a}$ \\
\hline & Zanthoxylum piperitum & $19.6(12.6-30.5)$ & $\mathrm{d}$ \\
\hline Saururaceae & Houttuynia cordata & $38.8(29.4-49.9)$ & $\mathrm{b}$ \\
\hline Saxifragaceae & Saxifraga stolonifera & $24.9(17.2-36.4)$ & $\mathrm{d}$ \\
\hline \multirow[t]{2}{*}{ Scrophulariaceae } & Digitalis purpurea & $15.5(11.9-20.0)$ & $\mathrm{d}$ \\
\hline & Paulownia tomentosa & $23.4(13.2-42.3)$ & $\mathrm{a}$ \\
\hline Smilacaceae & Smilax china & $11.4(7.36-17.5)$ & d \\
\hline Solanaceae & Brugmansia suaveolens & $25.2(16.2-39.4)$ & $\mathrm{d}$ \\
\hline
\end{tabular}




\section{Continued}

\begin{tabular}{|c|c|c|c|}
\hline \multirow[t]{6}{*}{ Solanaceae } & Lycium chinense & $15.1(10.9-20.7)$ & d \\
\hline & Physalis alkekengi var. franchetii & $57.4(43.9-70.9)$ & $\mathrm{d}$ \\
\hline & Solanum lyratum & $33.8(24.0-44.8)$ & $\mathrm{d}$ \\
\hline & Solanum nigrum & $33.9(19.0-62.6)$ & $\mathrm{b}$ \\
\hline & Solanum torvum & $12.9(9.15-18.0)$ & $\mathrm{c}$ \\
\hline & Withania somnifera & $6.96(5.56-8.68)$ & $\mathrm{c}$ \\
\hline Sterculiaceae & Brachychiton acerifolius & $29.6(22.6-38.7)$ & a \\
\hline Taxodiaceae & Cunninghamia lanceolata & $34.2(19.5-61.8)$ & $\mathrm{a}$ \\
\hline Trochodendraceae & Trochodendron aralioides & $17.1(11.7-25.1)$ & $\mathrm{a}$ \\
\hline \multirow[t]{3}{*}{ Verbenaceae } & Clerodendrum bungei & $31.1(23.9-39.1)$ & d \\
\hline & Lantana camara & $30.1(22.6-38.7)$ & $\mathrm{c}$ \\
\hline & Phyla canescens & $5.94(4.56-7.77)$ & $\mathrm{c}$ \\
\hline Zingiberaceae & Alpinia zerumbet & $20.9(17.4-25.1)$ & $\mathrm{c}$ \\
\hline
\end{tabular}

${ }^{1} \mathrm{EC}_{50}$ value unit; $\mathrm{mg}$ fresh weight equivalent $\mathrm{mL}^{-1}$ (95\% confidence interval); ${ }^{2}$ Sampling location: a, Tsukuba Botanical Garden, National Museum of Nature and Science, Tsukuba, Japan; b, National Institute for Agro-Environmental Sciences (NIAES), Tsukuba, Japan; c, Greenhouse in NIAES; d, Matsudo, Japan.

inhibitory activity against lettuce radicle elongation (estimated $\mathrm{EC}_{50}: 2.51 \mathrm{~F} . \mathrm{W}$. eq. $\mathrm{mL}^{-1}$ ). A detailed study was performed on the allelopathic potential of this species; the leaves of $T$. indica reportedly suppressed the growth of several weed and edible crop species [31]. The active compounds, however, have not yet been elucidated.

Albizia guachapele (Fabaceae; estimated $\mathrm{EC}_{50}: 3.08$ F.W. eq. $\mathrm{mL}^{-1}$ ) is one of the species selected as potential trees for reforestation in the study of Wishnie et al. [32]. Together with $A$. guachapele, G. sepium (estimated $\mathrm{EC}_{50}$ : 1.78 F.W. eq. $\mathrm{mL}^{-1}$ ) and $S$. saman (estimated $\mathrm{EC}_{50}: 2.20$ F.W. eq. $\mathrm{mL}^{-1}$ ) were also among their 24 selected species [32]. This is an unexpected correspondence because it would be reasonable that fast-growing trees expend their energy on photosynthesis and lignifications rather than the biosynthesis of secondary metabolites.

Indigofera kirilowii (Fabaceae; estimated $\mathrm{EC}_{50}: 3.08$ F.W. eq. $\mathrm{mL}^{-1}$ ) contains toxic glucose conjugates of 3-nitropropanoic acid in its roots [33]. The aerial parts of more than 60 species of Indigofera contain mono-, di-, tri-, and tetrasubstituted glucose esters of 3-nitropropanoic acid, which are toxic to livestock and other animals [34-36]. This implies that the plant growth-inhibitory components of I. kirilowii might be 3-nitropropanoic acid and/or its esters.

The estimated $\mathrm{EC}_{50}$ value of Caesalpinia pluviosa var. peltophoroides (Caesalpiniaceae) was 3.76 F.W. eq. $\mathrm{mL}^{-1}$. According to the literature, several Caesalpinia species have been studied phytochemically and pharmacologically. Cassane-type diterpenes [37-39], flavonoids [40, 41], biflavonoids [42], and tannins [43] have been iso- lated from Caesalpinia species.

Acacia podalyriifolia (Fabaceae; estimated $\mathrm{EC}_{50}: 4.75$ F.W. eq. $\mathrm{mL}^{-1}$ ) is native to Australia and is cultivated in southern Brazil as an exotic ornamental tree. Several species in the genus Acacia have important medicinal applications [44,45]. They possess phenolic compounds, for which countless biological activities are described $[45,46]$.

Kalmia latifolia (estimated $\mathrm{EC}_{50}$ : 4.79 F.W. eq. $\mathrm{mL}^{-1}$ ), a species of the Ericaceae family, is an evergreen understory shrub found in abundance on xeric southwest slopes in the southern Appalachian Mountains. It typically forms a dense understory in mixed pine/hardwood stands and occasionally in association with Rhododendron maximum [47,48]. Another species in this genus, $K$. angustifolia, contains toxic phenolic compounds [49], indicating that the activity of $K$. latifolia may be due to phenolics.

The results presented in this study provide essential information for further research on potential natural herbicides. For example, a series of studies have been conducted on cis-cinnamoyl derivatives isolated from $S$. thunbergii and S. prunifolia [50,51]. The chemical structure that is essential for the inhibitory activity is the cis-cinnamoyl moiety [19], and cis-cinnamic acid remains active after being mixed with alluvial soil under laboratory conditions [52]. Trials seeking to enhance the activity of cis-cinnamoyl derivatives by introducing functional groups and/or modifying the basic structure are in progress. This potential of $S$. thunbergii as a source of promising compounds was originally revealed 
in a screening study of 92 randomly selected tree species [18]. The list provided in the present paper (Table 1) could be useful to researchers who intend to find new lead compounds for natural herbicides.

\section{Acknowledgements}

This research was supported by the Programme for Promotion of Basic and Applied Researches for Innovations in Bio-oriented Industry (BRAIN).

\section{REFERENCES}

[1] N. G. Creamer, M. A. Bennett, B. R. Stinner and J. Cardina, "A Comparison of Four Processing Tomato Production Systems Differing in Cover Crop and Chemical Inputs," Journal of the American Society for Horticultural Science, Vol. 121, No. 3, 1996, pp. 559-568.

[2] S. A. Walters and B. G. Young, "Effect of Herbicide and Cover Crop on Weed Control in No-Tillage Jack-o-Lantern Pumpkin (Cucurbita pepo L.) Production," Crop Protection, Vol. 29, No. 1, 2010, pp. 30-33. doi:10.1016/j.cropro.2009.09.001

[3] B. J. Mazur and S. C. Falco, "The Development of Herbicide Resistant Crops," Annual Review of Plant Physiology and Plant Molecular Biology, Vol. 40, 1989, pp. 441-470. doi:10.1146/annurev.pp.40.060189.002301

[4] Z. L. Zhu and D. L. Chen, "Nitrogen Fertilizer Use in China-Contributions to Food Production, Impacts on the Environment and Best Management Strategies," Nutrient Cycling in Agroecosystems, Vol. 63, No. 2-3, 2002, pp. 117-127. doi:10.1023/A:1021107026067

[5] W. Zheng, S. R. Yates, S. K. Papiernik and M. Guo, "Transformation of Herbicide Propachlor by an Agrochemical Thiourea," Environmental Science and Technology, Vol. 38, No. 24, 2004, pp. 6855-6860. doi:10.1021/es049384+

[6] A. R. Putnam and C. S. Tang, "Allelopathy: State of the Science," In: A. R. Putnam \& C. S. Tang, Eds., The Science of Allelopathy, John Wiley \& Sons, New York, 1986, pp. 1-19.

[7] J. B. Lovett, "Changing Perceptions of Allelopathy and Biological Control," Biological Agriculture and Horticulture, Vol. 8, No. 2, 1991, pp. 89-100. doi:10.1080/01448765.1991.9754583

[8] H. P. Singh, D. R. Batish and R. K. Kohli, "Allelopathic Interactions and Allelochemicals: New Possibilities for Sustainable Weed Management," Critical Reviews in Plant Sciences, Vol. 22, No. 3-4, 2003, pp. 239-311. doi:10.1080/713610858

[9] G. Mitchell, D. W. Bartlett, T. E. Fraser, T. R. Hawkes, D. C. Holt, J. K.Townson and R. A.Wichert, "Mesotrione: A New Selective Herbicide for Use in Maize," Pest Management Science, Vol. 57, No. 2, 2001, pp. 120-128. doi:10.1002/1526-4998(200102)57:2<120::AID-PS254> 3.0.CO;2-E

[10] R. Beaudegnies, A. J. F. Edmunds, T. E. M. Fraser, R. G.
Hall, T. R. Hawkes, G. Mitchell, J. Schaetzer, S. Wendeborn and J. Wibley, "Herbicidal 4-Hydroxyphenylpyruvate Dioxygenase Inhibitors: A Review of the Triketone Chemistry Story from a Syngenta Perspective," Bioorganic and Medicinal Chemistry, Vol. 17, No. 12, 2009, pp. 4134-4152. doi:10.1016/j.bmc.2009.03.015

[11] Y. Fujii, S. S. Parvez, M. M. Parvez, Y. Ohmae and O. Iida, "Screening of 239 Medicinal Plant Species for Allelopathic Activity Using the Sandwich Method," Weed Biology and Management, Vol. 3, No. 4, 2003, pp. 233241. doi:10.1046/j.1444-6162.2003.00111.x

[12] Y. Fujii, T. Shibuya, K. Nakatani, T. Itani, S. Hiradate and M. M. Parvez, "Assessment Method for Allelopathic Effect from Leaf Litter Leachates," Weed Biology and Management, Vol. 4, No. 1, 2004, pp. 19-23. doi:10.1111/j.1445-6664.2003.00113.x

[13] C. I. M. Ono, R. Miyaura, M. L. T. Figueroa, E. L. R. Salgado and Y. Fujii, "Screening of 170 Peruvian Plant Species for Allelopathic Activity by Using the Sandwich Method," Weed Biology and Management, Vol. 12, No. 1, 2012, pp. 1-11. doi:10.1111/j.1445-6664.2011.00429.x

[14] Y. Fujii, "Screening of Allelopathic Candidates by New Specific Discrimination, and Assessment Methods for Allelopathy, and the Inhibition of L-DOPA [L-3,4-Dihydroxyphenylalanine] as the Allelopathic Substance from the Most Promising Velvetbean (Mucuna pruriens)," Bulletin of Natural Institute Agro-Environmental Sciences, Vol. 10, 1994, pp. 115-218. (in Japanese with English summary)

[15] Y. Fujii and T. Shibuya, "A New Assessment Method for Allelopathy by Agar Medium II. Mixed Culture of Alleopathic Condidates with Acceptor Plants in Agar Medium," Weed Research Japan, Vol. 36, 1991, pp. 152-153. (in Japanese)

[16] G. Hashimoto and Y. Nishimoto, "Illustrated Cyclopedia of Brazilian Medicinal Plants," Aboc-sha Press, Kamakura, 1996, p. 676.

[17] Y. Mimaki, H. Harada, C. Sakuma, M. Haraguchi, S. Yui, T. Kudo, M. Yamazaki and Y. Sashida, "Contortisiliosides A-G: Isolation of Seven New Triterpene Bisdesmosides from Enterolobium contortisiliquum and Their Cytotoxic Activity," Helvetica Chimica Acta, Vol. 87, No. 4, 2004, pp. 851-865. doi:10.1002/hlca.200490083

[18] S. Morita, M. Ito and J. Harada, "Screening of an Allelopathic Potential in Arbor Species," Weed Biology and Management, Vol. 5, No. 1, 2005, pp. 26-30. doi:10.1111/j.1445-6664.2005.00151.x

[19] S. Hiradate, S. Morita, A. Furubayashi, Y. Fujii and J. Harada, "Plant Growth Inhibition by cis-Cinnamoyl Glucosides and cis-Cinnamic Acid," Journal of Chemical Ecology, Vol. 31, No. 3, 2005, pp. 591-601. doi:10.1007/s10886-005-2047-0

[20] F. H. Utech and S. Kawano, "Floral Vascular Anatomy of Convallaria majalis L. and C. keiskei Miq. (LiliaceaeConvallariinae)," Journal of Plant Research, Vol. 89, No. 3, 1976, pp. 173-182. doi:10.1007/BF02488340

[21] R. Schrutka-Rechtenstamm, B. Kopp and W. Löffelhardt, "Bioconversions Leading to Minor Cardiac Glycosides in 
Convallaria majalis," Phytochemistry, Vol. 25, No. 5, 1986, pp. 1107-1109.

doi:10.1016/S0031-9422(00)81563-0

[22] P. Verron, M. Nard and J. Cohat, "In Vitro Organogenic Competence of Different Organs and Tissues of Lily of the Valley 'Grandiflora of Nantes'," Plant Cell Tissue and Organ Culture, Vol. 40, No. 3, 1995, pp. 237-242. doi:10.1007/BF00048129

[23] P. A. Chadhokar, "Gliricidia maculata: A Promising Legume Fodder Plant," World Animal Review, Vol. 44, 1982, pp. 36-43.

[24] L. E. Gale, M. R. Gibson and P. M. Scott, "Investigation of the Reported Toxicity to Rats of Gliricidia sepium, Jacq.," Science, Vol. 120, No. 3117, 1954, pp. 500-501. doi:10.1126/science.120.3117.500

[25] M. Ramamoorthy and K. Paliwal, "Allelopathic Compounds in Leaves of Gliricidia sepium (Jacq.) Kunth ex walp. and Its Effect on Sorghum vulgare L.," Journal of Chemical Ecology, Vol. 19, No. 8, 1993, pp. 1691-1701. doi:10.1007/BF00982301

[26] M. Tomita, T. Kikuchi, S. Uyeo, T. Nishinaga, M. Yasunishi and A. Yamamoto, "Pachysandra Alkaloids. I. Systematic Isolation and Characterization of Alkaloids," Yakugaku Zasshi, Vol. 87, No. 3, 1967, pp. 215-227. (in Japanese with English summary)

[27] S. Funayama, T. Noshita, K. Shinoda, N. Haga, S. Nozoe, M. Hayashi and K. Komiyama, "Cytotoxic Alkaloids of Pachysandra terminalis," Biological and Pharmaceutical Bulletin, Vol. 23, No. 2, 2000, pp. 262-264. doi: $10.1248 / \mathrm{bpb} .23 .262$

[28] R. N. Prasad, S. Viswanathan, J. R. Devi, V. Nayak, V. C. Swetha, B. R. Archana, N. Parathasarathy and J. Rajkumar, "Preliminary Phytochemical Screening and Antimicrobial Activity of Samanea saman," Journal of Medicinal Plants Research, Vol. 2, No. 10, 2008, pp. 268270.

[29] M. P. Raghavendra, S. Satish and K. A. Raveesha, "In Vitro Antibacterial Potential of Alkaloids of Samanea saman (Jacq.) Merr. against Xanthomonas and Human Pathogenic Bacteria," World Journal of Agricultural Sciences, Vol. 4, No. 1, 2008, pp. 100-105.

[30] M. Noor and M. A. Khan, "Allelopathic Potential of Albizia samans Merr.," Pakistan Journal of Botany, Vol. 26, 1994, pp 139-147.

[31] S. S. Parvez, M. M. Parvez, E. Nishihara, H. Gemma and Y. Fujii, "Tamarindus indica L. Leaf Is a Source of Allelopathic Substance," Plant Growth Regulation, Vol. 40, No. 2, 2003, pp. 107-115. doi:10.1023/A:1024237426416

[32] M. H. Wishnie, D. H. Dent, E. Mariscal, J. Deago, N. Cedeño, D. Ibarra, R. Condit and P. M. S. Ashton, "Initial Performance and Reforestation Potential of 24 Tropical Tree Species Planted across a Precipitation Gradient in the Republic of Panama," Forest Ecology and Management, Vol. 243, No. 1, 2007, pp. 39-49. doi:10.1016/j.foreco.2007.02.001

[33] Y. Su, C. Li, Y. Gao, L. Di, X. Zhang and D. Guo, “Acryloylated Glucose 3-Nitropropanoates from Indigofera kirilowii," Journal of Natural Products, Vol. 68, No. 12, 2005, pp. 1785-1786. doi:10.1021/np050268+

[34] W. S. Garcez, F. R. Garcez, N. K. Honda and A. J. R. da Silva, "A Nitropropanoyl-Glucopyranoside from Indigofera suffruticosa," Phytochemistry, Vol. 28, No. 4, 1989, pp. 1251-1252. doi:10.1016/0031-9422(89)80220-1

[35] V. Lodha, H. A. Khan and A. Ghanim, "5,7,4'-Trimethoxy-4-Phenylcoumarin from Roots of Indigofera oblongifolia," Journal of Indian Chemical Society, Vol. 75, No. 8, 1998, p. 485.

[36] M. Benn, D. Mcewan, M. A. Pass and W. Majak, "Three Nitropropanoyl Esters of Glucose from Indigofera linnaei," Phytochemistry, Vol. 31, No. 7, 1992, pp. 2393 2395. doi:10.1016/0031-9422(92)83284-6

[37] D. D. McPherson, C. T. Che, G. A. Cordell, D. D. Soejarto, J. M. Pezzuto and H. H. S. Fong, "Diterpenoids from Caesalpinia pulcherrima," Phytochemistry, Vol. 25, No. 1,1985 , pp. 167-170. doi:10.1016/S0031-9422(00)94522-9

[38] J. S. Roach, S. McLean, W. F. Reynolds and W. F. Tinto, "Cassane Diterpenoids of Caesalpinia pulcherrima," Journal of Natural Products, Vol. 66, No. 10, 2003, pp. 13781381. doi: $10.1021 / \mathrm{np} 0302955$

[39] S. Awale, T. Z. Linn, Y. Tezuka, S. K. Kalauni, A. H. Banskota, F. Attamimi, J. Ueda and S. Kadota, "Constituents of Caesalpinia crista from Indonesia," Chemical and Pharmaceutical Bulletin, Vol. 54, No. 2, 2006, pp. 213-218. doi:10.1248/cpb.54.213

[40] M. Namikoshi, H. Nakata and T. Saitoh, "Homoisoflavonoids from Caesalpinia sappan," Phytochemistry, Vol. 26, No. 6, 1987, pp. 1831-1833. doi:10.1016/S0031-9422(00)82298-0

[41] K. V. N. S. Srinivas, Y. K. Rao, I. Mahender, B. Das, K. V. S. R. Krishna, K. H. Kishore and U. S. N. Murty, "Flavanoids from Caesalpinia pulcherrima," Phytochemistry, Vol. 63, No. 7, 2003, pp. 789-793. doi:10.1016/S0031-9422(03)00325-X

[42] M. V. Bahia, J. B. dos Santos, J. P. David and J. M. David, "Biflavonoids and Other Phenolics from Caesalpinia pyramidalis (Fabaceae)," Journal of the Brazilian Chemical Society, Vol. 16, No. 6B, 2005, pp. 1402-1405. doi:10.1590/S0103-50532005000800017

[43] K. K. Awasthi, A. Kumar and K. Misra, "Two Ellagitannins from the Stem Bark of Caesalpinia pulcherrima," Phytochemistry, Vol. 19, No. 9, 1980, pp. 1995-1997. doi:10.1016/0031-9422(80)83020-2

[44] T. Sekine, N. Fukasawa, F. Ikegami, K. Saito, Y. Fujii and I. Murakoshi, "Structure and Synthesis of a New Monoterpenoidal Carboxiamide from the Seeds of the Thai Medicinal Plant Acacia concinna," Chemical and Pharmaceutical Bulletin, Vol. 45, No. 1, 1997, pp. 148-151. doi: $10.1248 / \mathrm{cpb} .45 .148$

[45] B. Meera, J. Chander and S. B. Kalidhar, "A Review on the Chemistry and Bioactivity of the Acacia spp.," Journal of Medicinal and Aromatic Plant Sciences, Vol. 27, 2005, pp. 51-90.

[46] V. A. Kerber and G. A. A. B. Silva, "Flavonóides da 
Acacia longifolia (Andr.) Willd.-Leguminosae-Mimosoideae," Revista Brasileira de Farmacognosia, Vol. 74, No. 1, 1993, pp. 16-18.

[47] M. V. Lipscomb and E. T. Nilsen, "Environmental and Physiological Factors Influencing the Natural Distribution of Evergreen and Deciduous Ericaceous Shrubs on Northeast- and Southwest-Facing Slopes of the Southern Appalachian Mountains. II. Water Relations," American Journal of Botany, Vol. 77, No. 4, 1990, pp. 517-526. doi: $10.2307 / 2444386$

[48] B. D. Clinton, L. R. Boring and W. T. Swank, "Regeneration Patterns in Canopy Gaps of Mixed-Oak Forests of the Southern Appalachians: Influences of Topographic Position and Evergreen Understory," American Midland Naturalist, Vol. 132, No. 2, 1994, pp. 308-319. doi: $10.2307 / 2426587$

[49] H. Zhu and A. U. Mallik, "Interactions between Kalmia and Black Spruce: Isolation and Identification of Allelopathic Compounds," Journal of Chemical Ecology, Vol. 20, No. 2, 1994, pp. 407-421. doi:10.1007/BF02064447

[50] S. Hiradate, S. Morita, H. Sugie, Y. Fujii and J. Harada, "Phytotoxic cis-Cinnamoyl Glucosides from Spirea thunbergii," Phytochemistry, Vol. 65, No. 6, 2004, pp. 731739. doi:10.1016/j.phytochem.2004.01.010

[51] S. Morita, S. Hiradate, Y. Fujii and J. Harada, "cis-Cinnamoyl Glucoside as a Major Plant Growth Inhibitor Contained in Spiraea prunifolia," Plant Growth Regulation, Vol. 46, No. 2, 2005, pp. 125-131. doi:10.1007/s10725-005-8086-2

[52] S. Hiradate, K. Ohse, A. Furubayashi and Y. Fujii, "Quantitative Evaluation of Allelopathic Potentials in Soils: Total Activity Approach," Weed Science, Vol. 58, No. 3, 2010, pp. 258-264. doi:10.1614/WS-D-09-00085.1 